\title{
E-PAP: Enhanced Priority Aware Protocol for Reliable Communication in Wireless Body Area Network
}

\author{
Archana Sandhu, Amita Malik
}

\begin{abstract}
Wireless Body Area Network (WBAN) is a collection of miniaturized sensing nodes and coordinator nodes. These sensing nodes are placed in, on and around the body for uninterrupted monitoring of physiological data for medical applications. The main application carrier of WBAN is the human body and due to human body movement and physiological changes, the WBAN traffic fluctuates greatly. This network traffic fluctuation requires good network adaptability. In addition to traffic fluctuations, energy consumption is another key problem with WBANs as sensing nodes are very small in size. This paper design a reliable protocol by extending the MAC protocol for reducing energy consumption, PAP algorithm to decide data transmission rate and JOAR algorithm to select the optimize path for the data transmission. The performance of the algorithm outperforms other state of art algorithms to shows its significance.
\end{abstract}

Keywords: Reliable, PAP, Jaya, WBAN, Energy Efficiency

\section{INTRODUCTION}

Wireless Body Area Networks (WBAN) have recently attracted a wide interest of biomedical applications to realize undiscovered fields of medical services. This fusion of communication technologies and medical services can contribute to the wide distribution of medical knowledge and conserve the cost of medical services. In WBANs, a communication network centering on human body is created by connecting micro-sized sensors attached to human body and communication devices (e.g. mobile phones, PDA etc.) around the body [1]. This setup is able to monitor and collect physiological signals such as body temperature, blood pressure, heart rate, electrocardiogram (ECG) and electroencephalogram (EEG) etc. along with body motion and surrounding environment [2]. These signals are to be forwarded to remote monitoring centers with high efficiency of energy utilization and minimized latency for emergency data whenever a node senses any critical signal about human body. Coordinator nodes are responsible for analyzing the sensed data, synchronizing the sensing nodes against collisions and interferences and transmitting data to remote monitoring centers with efficient energy utilization [3].

Revised Manuscript Received on August 15, 2020.

* Correspondence Author

Archana*, Department of Computer Applications, Deenbandhu Chotu Ram University of Science and Technology, Murthal, Sonepat, Haryana, India.

Dr. Amita Malik, Professor and Chairperson, Department of Computer Science \& Engineering, Deenbandhu Chotu Ram University of Science and Technology, Murthal, Sonepat, Haryana, India.

(c) The Authors. Published by Blue Eyes Intelligence Engineering and Sciences Publication (BEIESP). This is an open access article under the CC BY-NC-ND license (http://creativecommons.org/licenses/by-nc-nd/4.0/)
A coordinator has relatively sufficient energy than small sized sensing nodes as it has rechargeable batteries and other hardware resources. Therefore, there should be an energy balance between the coordinator and sensing nodes. WBANs are widely used in sports, healthcare and telemedicine etc. [4]. In WBAN, medium access control (MAC) layer plays an important role in efficient energy usage and reliable communication as it deals with processing and synchronization of nodes, conflict detection and priority control. Hence, the performance of WBAN is closely dependent on MAC layer protocol [5]. The WBAN environment can be closely analyzed by analyzing the MAC layer. For example, an uplink direction indicates that the data is transmitted mostly from nodes to coordinator and data can be classified as periodic and emergency data to provide priority-based treatment of data. For efficient energy usage, the MAC protocol has to adaptively and asymmetrically manage the energy balance between the coordinator and sensing nodes. Various MAC protocols have been proposed and designed for wireless sensor networks, but these cannot be directly applied to WBAN due to its unique features and requirements as discussed above. Hence, there is a need of MAC protocol that can provide an energy balance between the sensing nodes and coordinator and also provide minimum transmission delay for emergency data. These MAC protocol issues lead us to design our proposed protocol. The protocol will improve the performance in terms of latency, energy consumption and throughput. The remaining paper is organized in following sections: Section 2 discusses existing MAC protocols; Section 3 describes the working of proposed protocol, while the proposed MAC protocol is compared with IEEE 802.15.6 MAC protocol and evaluated using network simulator NS2 in Section 4. Section 5 gives the conclusions.

\section{RELATED WORK}

The specially designed communication standard for WBAN is IEEE 802.15.6 [6]. This communication standard has given an energy efficient solution on both MAC and Physical (PHY) layers and employed a combined approach of slot-allocation and random-access. The superframe structure as shown in figure 1 is classified into nine different phases [7] namely: beacon phase (B), exclusive access phase one (EAP1), random access phase one (RAP1), managed access phase one (MAP1), exclusive access phase two (EAP2), random access phase two (RAP2), managed access phase two (MAP2), beacon phase two (B2) and contention access phase (CAP).

Published By:

Blue Eyes Intelligence Engineering

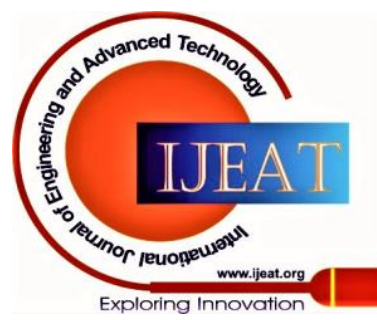


Reserved data of nodes is transmitted through slot-assigned period without any contention. EAP and RAP are used by the nodes for non-reserved emergency data transmission. The non-reserved emergency transmission of data only through EAP and RAP is the main limitation of this protocol. B. Liu et al. [8] proposed a context aware MAC protocol which deploys a hybrid superframe structure based on traffic nature and channel status. This protocol defines access policies according to channel sensing and allocates time slots based on traffic awareness. C. L. Fourati et al. [9] proposed a priority-based hybrid MAC protocol where the transmission channel is divided into data channel and control channel and priority is assigned to health critical information. Sleep mode is used for increased network lifetime. In [10], a priority-based MAC protocol is defined where, emergency data is transmitted during non contention access period and periodic data is transmitted through contention access period. The coordinator node allocates time slots to other sensing nodes according to their priority which results in reduced collision and hence, reduced energy consumption and transmission delay. S. Rezvani et al. [11] proposed a MAC protocol where, data rate of node is increased by allocating resources as per the condition of network which results in an efficient and energy saving protocol (H-MAC). In [12], an adaptive MAC protocol is defined, which uses Time Division Multiple Access (TDMA) to access the channel and a synchronization scheme is used for collision avoidance. M. A. Huq et al. [13] proposed a Medical Emergency MAC protocol, which uses idle time slot to insert an additional listening window for emergency services without affecting network throughput by defining long superframe for emergency data access to minimize the delay. Li. H. et al. [14] proposed H-MAC, which is a TDMA based MAC protocol. However, this protocol uses heartbeat vibration as synchronization tick for TDMA instead of receiving any beacon frame from the coordinator. Therefore, all nodes transmit data without any contention assuming that they already had the sequence for slot assignment. BodyMAC [15], an IEEE 802.15.4 based protocol cut off the inactive period from IEEE 802.15.4 and introduces a newly assigned downlink subframe in place of CFP in superframe. All the sensor nodes are guaranteed to be assigned to downlink slots to save energy from idle. The coordinator uses a wake-up signal transmission process to activate a sleeping implant. The activated implant synchronizes to the wake-up signal and find next beacon frame to receive data from coordinator.

JOAR [16], given by same author uses the Jaya based optimization to select the optimized route for transmission on the basis of specific absorption ratio (SAR) and the duty cycle. The optimized route selected by JOAR algorithm outperforms the other state of art algorithm i.e. Anthocnet, AODV and DSDV in terms of delay, packet delivery ratio and throughput. PAP [17] decides the data transmission rate of each packet and the sensing interval of each node on the basis of dynamic and the static priorities. The static priority dependents upon the functionality of the node while the dynamic priority depends upon the data value range being measured. The PAP algorithm performs better in terms of throughput and delay while the energy consumption of the algorithm is higher due to high data transmission rate. This paper improves the energy consumption of PAP by extending the MAC discussed in next section.

\section{PROPOSED WORK}

This reliability of any protocol depends upon the emergency data transferring priority, rate at which the data is transmitted and the routing path of the data. This paper considers all above concept along with the sensing interval and the energy consumption by the nodes. This work firstly checks the node type as shown in the figure 1 to decide the frame structure of the packet. The coordinator node has two phases i.e. Active phase and the sleep phase and uses the Sync to synchronization and CP for the contention period of other nodes. Remaining nodes has a beacon interval to update the priority of node with the coordinator node. The contention period of the node is itself divided into three subphases for requesting, receiving and

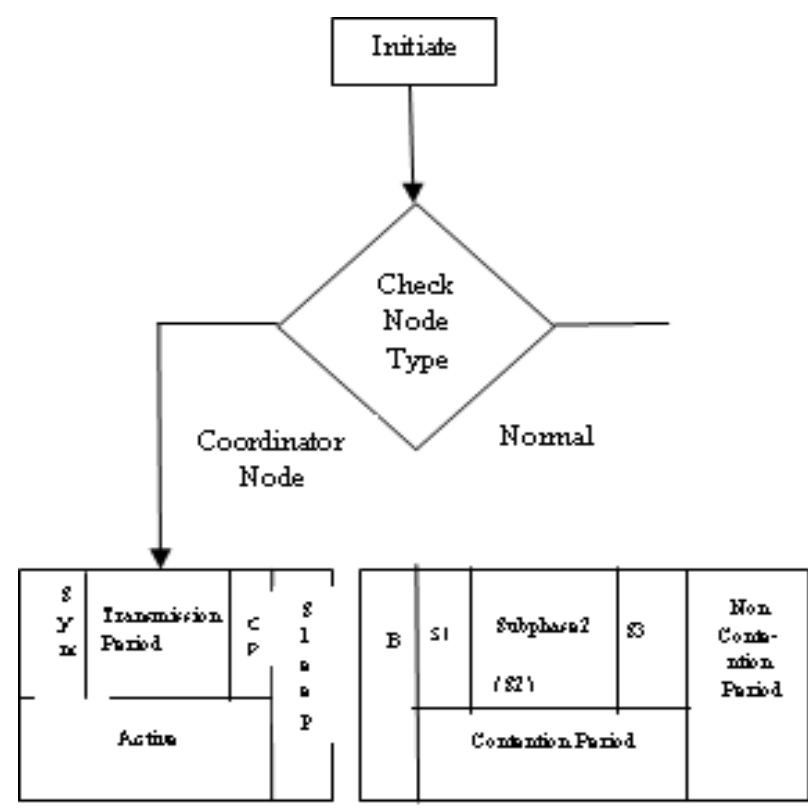

Fig 1: Frame Structure

acknowledgement of the data. The figure 1 shows the frame structure of the packet at coordinator node and the remaining nodes. The sleep node in the coordinator node is used for reducing the energy consumption while the content period of the node is decided on the basis of priority of the data by the coordinator node as shown in the figure 2 . The transmission rate and the sensing interval of the nodes are decided on the basis of PAP algorithm [17]. The data is transmitted at the specified rate with identified sensing interval described in figure 2. The normal node process and dispatch the unicast request to the coordinator node. On receiving the ACK from the coordinator node, normal node sends ACK as shown in figure 2. Then the JOAR algorithm [16] is used to get the optimized path on the basis of duty cycle of the node along and specific absorption rate. This process makes this protocol a reliable protocol due to reduced energy consumption by the sleep mode, instant transmission of emergency data at high data rate with high priority due to PAP algorithm (to decide the data transmission rate)

Published By:

Blue Eyes Intelligence Engineering and Sciences Publication (C) Copyright: All rights reserved. 
and the contention period time on the basis of priority and transmission of data through the optimized path by using the JOAR algorithm on the basis of duty cycle and SAR. The protocol has been implemented using NS2 and analyzed over different scenarios discussed in next section.

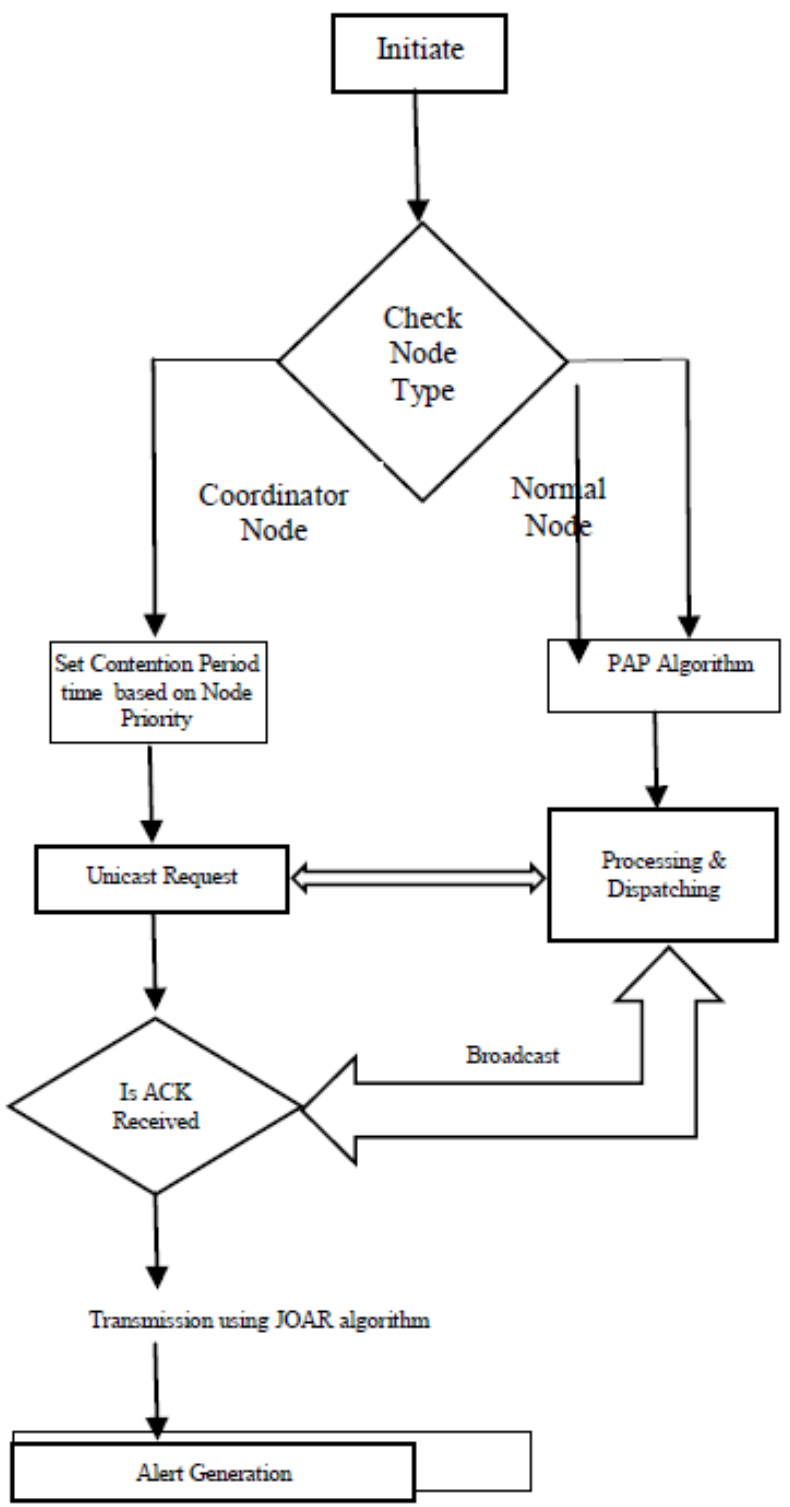

Figure 2: Flowchart for Reliable Protocol

IV. RESULT AND DISCUSSION

The proposed algorithm has been implemented using the network simulator. The analysis has been done on the same scenario as used by the PAP algorithm [17]. The analysis is done on a scenario having 7 nodes with one coordinator node. The nodes has low range of $5 \mathrm{~m}$ while covers the area of $0.4 * 0.4 \mathrm{~m}^{2}$. The performance of proposed protocol is analyzed on the basis of three parameters i.e. Throughput, End-to-End Delay and Remaining Energy.

\section{Throughput}

Figure 3 shows the comparison of PAP throughput on different networks having 1000, 2000, 3000, 4000, 5000 seconds as the simulation time. In each scenario the throughput for proposed protocol is higher as compared to other protocols.

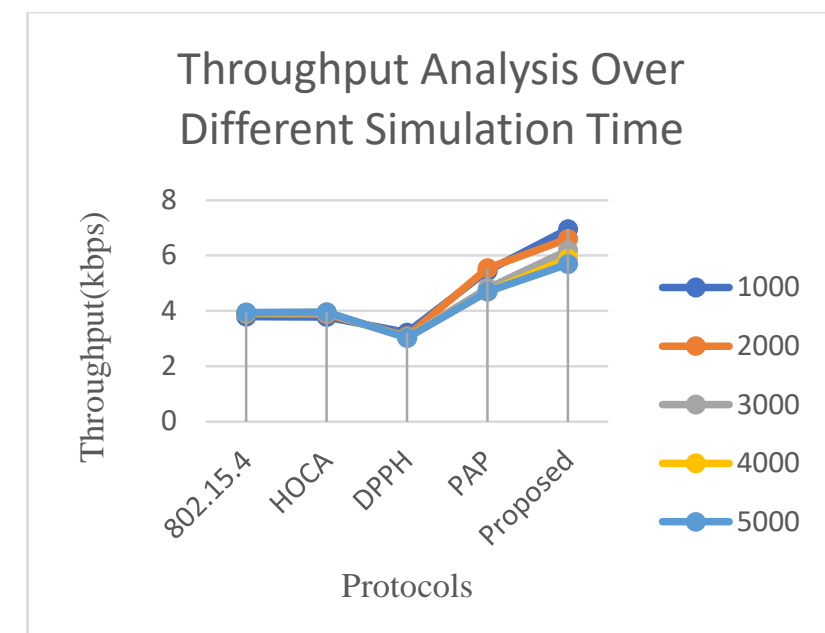

Figure 3: Throughput analysis over Different Simulation Time

The comparison clearly shows that proposed protocol outperforms the other protocols as the throughput is increased in each scenario as compared to other state of art techniques.

\section{End-to-End Delay}

Figure 4 compares the proposed protocol end-to-end delay with PAP, DPPH, 802.15.4 and HOCA for the scenario having 1000, 2000, 3000, 4000 and 5000 seconds simulation time. The decreased in the delay shows the better reliability of the protocol as compared to other protocols.

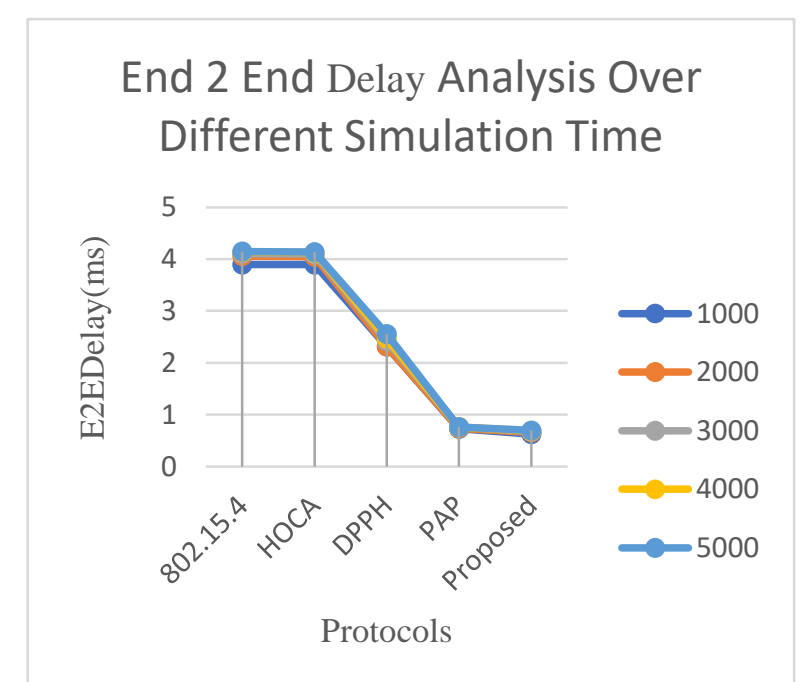

Figure 4: E2Edelay analysis over Different Simulation Time

\section{Remaining energy}

Figure 5 shows that higher energy consumption by the PAP protocol is removed by the proposed protocol by using the sleep phase.

Blue Eyes Intelligence Engineering

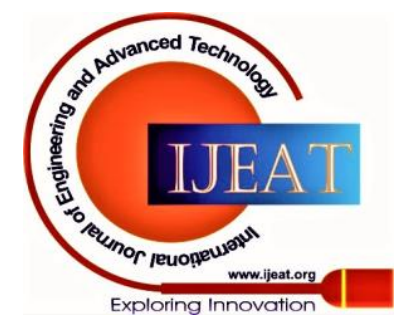




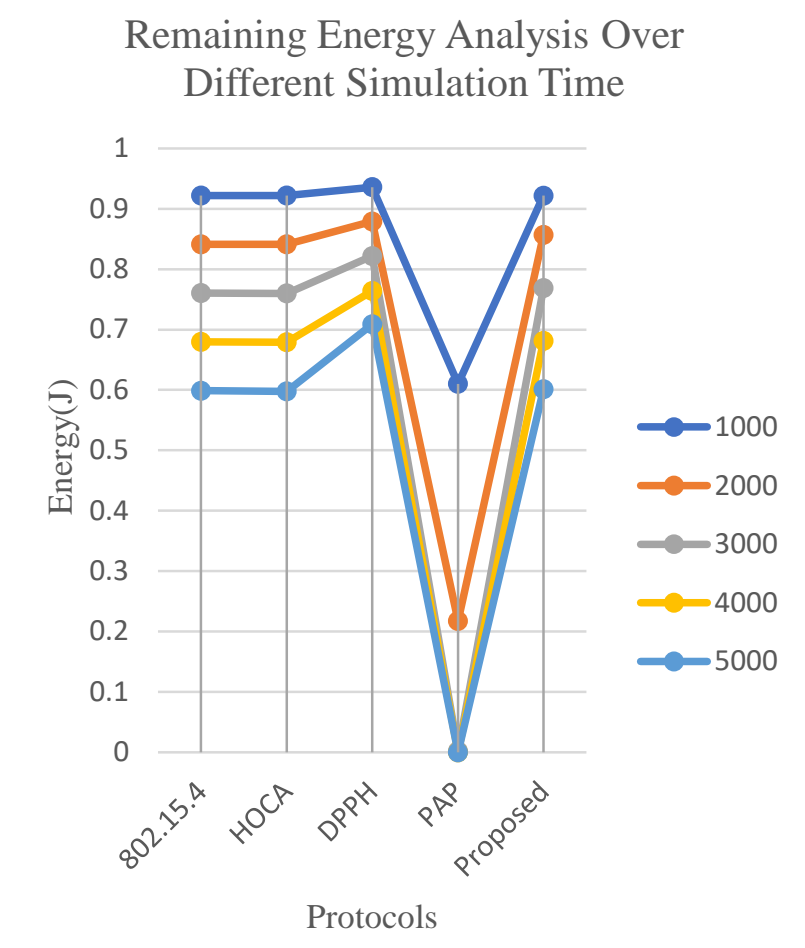

Figure 5: Remaining Energy analysis over Different Simulation Time

The comparison in figure 5 clearly signifies that protocol exhibits better performance as compared to all other existing protocols.

\section{CONCLUSION}

This paper design a reliable protocol by extending the MAC, using the PAP algorithm and the JOAR algorithm. The extended Mac reduces the energy consumption by using sleep mode and transmits emergency data by using contention period. The data transmission rate and the optimized routing path is selected by using the PAP and JOAR algorithm respectively. The performance of the proposed work is compared with the PAP, HOCA, DPPH and standard MAC i.e. 802.15 .4 by using the delay, throughput and remaining energy as parameters. The proposed protocol performs better as compared to all the other existing protocol and proves its significance. In future this protocol can be tested on real test-beds.

\section{REFERENCES}

1. IEEE 802.15.6: Wireless Body Area Networks. IEEE Standard for Local and Metropolitan Area Networks. 2012. Available online: http://ieeexplore.ieee.org/document/6161600 (accessed on 13 January 2020).

2. O. Gama and R. Simoes, "A Hybrid MAC scheme to improve the transmission performance in body sensor networks," Wireless Personal Communications, vol. 80, no. 3, pp. 1263-1279, 2014.

3. M. Chen, S. Gonzalez, A. Vasilakos, H. Cao, and V. C. M. Leung, "Body area networks: a survey," Mobile Networks and Applications, vol. 16, no. 2, pp. 171-193, 2011.

4. Y. Liu, Research on Key Technologies of Wireless Body Area Network, Beijing University of Posts and Telecommunications, 2017.

5. R. K. Bedi, "An improved energy efficient TDMA based MAC protocol for WBAN," International Journal of Computerences \& Engineering, vol. 6, no. 3, pp. 34-39, 2018.

6. Y. T. Xie, J. Wang, J. M. Min, and G. J. Si, "A highly-efficient energy-saving wireless body area network MAC protocol," Computer Technology and Development, vol. 25, no. 12, pp. 91- 96, 2015.
7. M. Bukvic and J. Misic, "Access anomaly of emergency traffic in CSMA/CA of IEEE 802.15.6," in Proceedings of the 2015 International Wireless Communications and Mobile Computing Conference (IWCMC), pp. 491-496, Dubrovnik, Croatia, August 2015.

8. B. Liu, Z. S. Yan, and C. W. Chen, "MAC protocol in wireless body area networks for E-health: challenges and a context- aware design,' IEEE Wireless Communications Magazine, vol. 20, no. 4, pp. 64-72, 2013.

9. C. L. Fourati, S. Boudjit, and L. Kamoun, "New priority MAC protocol for wireless body area networks," in Proceedings of the ACM MOBIHOC Workshop on Pervasive Wireless Healthcare, pp. 1-6, ACM, 2013.

10. J. Zhou, A. H. Guo, J. Xu, B. Celler, and S. Su, "A reliable medium access mechanism based on priorities for wireless body sensor networks," in Proceedings of the International Conference of the IEEE Engineering in Medicine \& Biology Society IEEE Engineering in Medicine \& Biology Society Conference, p. 1855, 2013.

11. S. Rezvani and S. A. Ghorashi, "A novel WBAN MAC protocol with improved Energy consumption and data rate," Ksii Trans- actions on Internet \& Information Systems, vol. 6, no. 9, pp. 2302- 2322, 2012.

12. N. Javaid, Ahmad A., and A. Rahim, "Adaptive medium access control protocol for wireless body area networks," International Journal of Distributed Sensor Networks, vol. 10, no. 3, pp. 156-160, 2014.

13. M. A. Huq, E. Dutkiewicz, G. Fang, R. P. Liu, and R. Vesilo, "MEB MAC: improved channel access scheme for medical emergency traffic in WBAN," in Proceedings of the International Symposium on Communications and Information Technologies (ISCIT '12), pp. 371376, IEEE, Queensland, Australia, October 2012.

14. [14] Li, H.; Tan, J. Heartbeat Driven Medium Access Control for Body Sensor Networks. HealthNet 2007, 14, 25-30.

15. Fang, G.; Dutkiewicz, E. BodyMAC: Energy efficient TDMA-based MAC protocol for wireless, body area networks. In Proceedings of the 9th IEEE International Symposium on Communications and Information Technology ISCIT, Icheon, Korea, 28-30 September 2009; pp. 1455-1459.

16. Sandhu A., Malik A. PAP: JOAR: Jaya Based Optimized and Reliable Protocol for Wireless Body Area Network. 'International Journal of Innovative Technology and Exploring Engineering (IJITEE)' that will publish at Volume-9 Issue-3.

17. Sandhu A., Malik A. PAP: Priority Aware Protocol for Healthcare Applications in Wireless Body Area Network. 'International Journal of Recent Technology and Engineering (IJRTE)' that will publish at Volume-8 Issue-5, January 2020

\section{AUTHOR PROFILE}

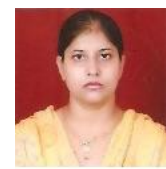

Archana received the $\mathrm{M}$.Tech. degree in software engineering in 2010 and pursuing Ph.D degree in computer applications from Deenbandhu Chotu Ram University of Science and Technology, Murthal, Sonepat. Her research areas are Wireless Sensor Network, Body Area Network. She is a lifetime member of ISTE. Her publications include about 9 research papers in given areas.

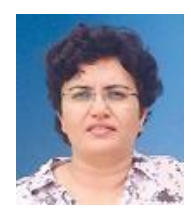

Dr. Amita Malik received her Ph.D degree in Computer Science \& Engineering from NIT, Kurukshetra. Her research areas are Mobile Ad hoc and Wireless sensor networks, Cloud Computing and Speech Processing. She is a lifetime member of ISTE and CSI. Her publications include 52 international and national research papers. She is currently Professor and Chairperson of Computer Science \& Engineering Department in Deenbandhu Chotu Ram University of Science and Technology.

Published By:

Blue Eyes Intelligence Engineering

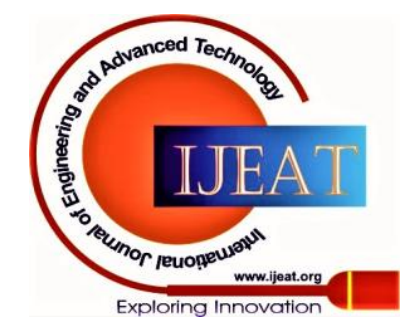

